Mon. Not. R. Astron. Soc. 000, 15 (2015) Printed 9 August $2021 \quad$ (MN LATEX style file v2.2)

\title{
Extragalactic magnetic fields unlikely generated at the electroweak phase transition
}

\author{
Jacques M. Wagstaff* and Robi Banerjee \\ Hamburger Sternwarte, Gojenbergsweg 112, 21029 Hamburg, Germany
}

\begin{abstract}
In this letter we show that magnetic fields generated at the electroweak phase transition are most likely too weak to explain the void magnetic fields apparently observed today unless they have considerable helicity. We show that, in the simplest estimates, the helicity naturally produced in conjunction with the baryon asymmetry is too small to explain observations, which require a helicity fraction at least of order $10^{-14}-10^{-10}$ depending on the void fields constraint used. Therefore new mechanisms to generate primordial helicity are required if magnetic fields generated during the electroweak phase transition should explain the extragalactic fields.
\end{abstract}

Key words: magnetic fields - MHD - early Universe.

\section{INTRODUCTION}

The evolution of magnetic fields depends strongly on its helicity. On the one hand, the global conservation of magnetic helicity directly leads to an inverse cascade of energy from small scales to large scales e.g. (Biskamp 1993, Christensson et al. 2001, Banerjee \& Jedamzik 2004). On the other hand, the helicity conservation leads to slower decay of the magnetic energy compared to the non-helical case, potentially producing stronger fields at present day. This combination of effects could prove to be of great importance for the explanation of large scale magnetic fields observed in the Universe today (Banerjee \& Jedamzik 2004, Tashiro et al. 2013). In this short note we aim to constrain the primordial magnetic helicity from the apparent observations of void magnetic fields. We show that magnetic fields generated at the electroweak phase transition (EWPT) must have considerable helicity in order to explain the extragalactic magnetic fields and that the helicity density arising naturally with electroweak baryogenesis is too weak to be the dominant source of magnetic helicity (see (Sigl 2002) for an early discussion on this topic). Our conclusions are based on well established results from magnetohydrodynamic (MHD) turbulence for the magnetic field decay rates (Banerjee \& Jedamzik 2004 Campanelli 2007a 2014) and the simplest estimates of magnetic helicity generation by (Vachaspati 2001). Any deviations from these conclusions would require deviations from such simple assumptions (see e.g. (Kahniashvili et al. 2013 Brandenburg et al. 2015), which we discuss later in this work.

* jwagstaff@hs.uni-hamburg.de

\section{BASIC EQUATIONS}

We start by writing down the two-point correlation function for a statistically homogeneous and isotropic stochastic magnetic field (see e.g. (Durrer \& Neronov 2013) and references therein)

$$
\begin{aligned}
\left\langle B_{i}(\boldsymbol{k}) B_{j}^{*}\right. & \left.\left(\boldsymbol{k}^{\prime}\right)\right\rangle=(2 \pi)^{3} \delta\left(\boldsymbol{k}-\boldsymbol{k}^{\prime}\right) \times \\
\times & {\left[\left(\delta_{i j}-\hat{k}_{i} \hat{k}_{j}\right) P_{B}(k)+i \epsilon_{i j k} \hat{k}_{k} P_{H}(k)\right] . }
\end{aligned}
$$

The above spectrum has been decomposed into parity conserving and parity violating components, or magnetic and helical spectra. The magnetic spectrum is given by $P_{B}(k)=\left\langle|\boldsymbol{B}(\boldsymbol{k})|^{2}\right\rangle / 2 \equiv\left(\rho / k^{2}\right)\left\langle M_{k}\right\rangle$, which depends only on the amplitude of $\boldsymbol{k}$ not its direction. We define the spectrum $M_{k}$ above to match the conventions of (Saveliev et al. 2012, 2013), where $\rho$ is the total energy density and comoving quantities are used throughout. Then, assuming a power law $P_{B}(k) \simeq P_{B}^{0} k^{n}$ on large scales, we can volume average on a region of size $L^{3}$ to estimate the average field on a given scale $L$ (Durrer \& Caprini 2003)

$$
B_{L}^{2} \equiv\left\langle\boldsymbol{B}_{L}^{2}(\boldsymbol{x})\right\rangle=\frac{P_{B}^{0}}{2 \pi^{2}} \frac{1}{L^{n+3}} \Gamma\left(\frac{n+3}{2}\right) .
$$

Causality restricts the power law index $n$ to be an even integer $n \geqslant 2$ (Durrer \& Caprini 2003). For the limiting case $n=2$, which is the expected scaling, one finds that $B_{L} \propto L^{-5 / 2}$. In (Saveliev et al. 2012) the authors showed numerically that, independent of the turbulent flow, a large scale magnetic field tail develops with the scaling $B_{L} \sim L^{-5 / 2}$, in agreement with the causality constrained averaged field described above.

The averaged magnetic energy density is obtained by 
integrating over the local energy density $u_{B}=\boldsymbol{B}^{2} / 8 \pi$, i.e.

$$
\epsilon_{B}=\frac{1}{V} \int u_{B} \mathrm{~d} \boldsymbol{r}=\frac{1}{8 \pi} \int|\boldsymbol{B}(\boldsymbol{k})|^{2} \mathrm{~d} \boldsymbol{k}=\rho \int M_{k} \mathrm{~d} k .
$$

Assuming that the magnetic energy is concentrated at the integral scale (denoted by the index ' $I$ '), which is the peak of the spectrum in Fourier space, we can write $\epsilon_{B}=\rho \int k M_{k} \mathrm{~d} \ln k \simeq \rho k_{I} M_{I}$. In the above we adopt the conventions from (Saveliev et al. 2012), where the authors showed numerically that indeed most of the energy is concentrated at the integral scale. We can also define an effective magnetic field strength $\epsilon_{B}=\int \mathrm{d} \ln k\left(B_{k}^{\text {eff }}\right)^{2} / 8 \pi$. In the above we set the wavenumber $k=2 \pi / L$ corresponding to the scale $L$ in Eq. (2). We can also identify the integral scale as the coherence length $\left(\lambda_{B}\right)$, and the effective magnetic field as the observed magnetic field strength $\left(B_{\lambda}\right)$, hence $M_{I}=B_{I}^{2} / 8 \pi \rho k_{I}=B_{\lambda}^{2} \lambda_{B} / 16 \pi^{2} \rho$.

The helical part of the spectrum is determined by

$$
P_{H}(k)=-\frac{i}{2}\left\langle(\hat{\boldsymbol{k}} \times \boldsymbol{B}(\boldsymbol{k})) \cdot \boldsymbol{B}^{*}(\boldsymbol{k})\right\rangle \equiv-\frac{\rho}{8 \pi k}\left\langle\mathcal{H}_{k}\right\rangle,
$$

where again the helical spectrum $\mathcal{H}_{k}$ is defined following the conventions of (Saveliev et al. 2013). On any given scale $k$ there is a realizability condition given by $\left|\mathcal{H}_{k}\right| \leqslant 8 \pi M_{k} / k$, from which we can define $f \equiv k \mathcal{H}_{k} / 8 \pi M_{k}$ as the helicity fraction, where $f=0$ for the non-helical case and $f=1$ for the maximally helical case. The average helicity density is given by

$$
h_{B}=\frac{1}{V} \int(\boldsymbol{A} \cdot \boldsymbol{B}) \mathrm{d} \boldsymbol{r}=\rho \int \mathcal{H}_{k} \mathrm{~d} k \simeq \rho k_{I} \mathcal{H}_{I},
$$

where $\boldsymbol{B}=\nabla \times \boldsymbol{A}$, and in the last equality we also assume that the helicity density is concentrated at the integral scale. Magnetic helicity is a useful quantity since it is conserved $h_{B} \simeq$ const. in the early Universe when the conductivity is very large (Biskamp 1993).

\section{BASIC CONSTRAINTS}

Let us first consider the constraints on void magnetic fields from the $\gamma$-ray observations of $\mathrm{TeV}$ Blazars (Neronov \& Vovk 2010, Taylor et al.|2011; Tavecchio et al. 2010, Dolag et al. 2011, Essey et al. 2011, Dermer et al. 2011). Authors in (Taylor et al. 2011) showed that the minimum magnetic field strength depends on the mechanism of suppression of the cascade signal. For suppression due to time delay, the minimum required field strength is $\sim 10^{-17} \mathrm{G}$, whereas for the extended emission they find $\sim 10^{-15} \mathrm{G}$ (see also (Tavecchio et al. 2010, Essey et al. 2011; Dermer et al. 2011)). The above bounds become tighter as $\lambda_{B}^{-1 / 2}$ for scales smaller than $\sim 1$ Mpc. We note that the above observations are not conclusive (Arlen et al. 2012 Broderick et al. 2012), and the authors in (Taylor et al. 2011) stress that, in any case, the bounds should be taken as an order-of-magnitude estimate. However, in this work we assume that the above constraints are actual bounds on void magnetic fields. For the purpose of this work we shall consider the bounds (Taylor et al. 2011 Dermer et al. 2011)

$$
B_{\lambda} \gtrsim\left(10^{-15}-10^{-18}\right) \lambda_{B}^{-\frac{1}{2}} \mathrm{G}, \quad \lambda_{B} \lesssim 1 \mathrm{Mpc},
$$

and later comment on our conclusions if this bound is relaxed somewhat.
The second constraint comes from energy considerations. The initial magnetic energy at magnetogenesis (denoted by the index ' $*$ ') can at most be in equipartition with radiation, i.e. $u_{B}=\rho / 2$, hence

$$
B_{\lambda, *} \leqslant B_{\lambda, *}^{\max } \equiv(4 \pi \rho)^{\frac{1}{2}} \simeq 3 \times 10^{-6} \mathrm{G},
$$

where the radiation here is taken to be the CMB photons (Banerjee \& Jedamzik 2004). We note that this bound satisfies the constraint from Big Bang Nucleosynthesis (Kahniashvili et al. 2011).

For magnetic fields generated at a time during the radiation dominated era (in contrast to inflationary magnetogenesis), the basic constraint on the coherence length is the horizon size at the time of magnetogenesis

$$
\lambda_{B, *} \leqslant\left.\lambda_{B, *}^{\max } \equiv \frac{1}{a H}\right|_{*} .
$$

The horizon size is $2 \times 10^{-10} \mathrm{Mpc}$ and $3 \times 10^{-7} \mathrm{Mpc}$ at the electroweak and QCD phase transitions respectively.

For the purpose of this paper, these are the only constraints that we need to consider.

\section{MAGNETIC FIELD EVOLUTION}

The magnetic field strength and coherence length evolve during the radiation dominated era due to turbulent MHD effects (Sigl 2002, Banerjee \& Jedamzik 2004 Campanelli 2007b; Durrer \& Neronov 2013. Wagstaff et al. 2014). Such effects include the free turbulent decay of magnetic fields, which is what we are mostly concerned with here, but we note that the turbulent amplification of weak fields by the small-scale dynamo is also possible (Wagstaff et al. 2014). In this paper we quote well known results from MHD turbulence and in particular we use the decay laws from the detailed studies in (Banerjee \& Jedamzik 2004 Campanelli 2007a, 2014, Saveliev et al. 2012, 2013).

If the initial spectral helicity is negligible $f_{*} \ll 1$, there is a direct cascade of energy due to the selective decay of modes in $k$-space. Indeed, the large $k$-modes are dissipated and decay so that the integral scale evolves down along the large scale spectrum. The general decay law for the magnetic energy is $M_{I} \propto t^{-2\left(n^{\prime}-1\right) /\left(n^{\prime}+2\right)}$ and for the integral scale $L_{I} \propto t^{2 /\left(n^{\prime}+2\right)}$, where $n^{\prime} \equiv n+3$. These decay laws are obtained through analytical considerations in (Banerjee \& Jedamzik 2004, Saveliev et al. 2012, 2013, Campanelli 2007a 2014) and confirmed numerically in (Banerjee \& Jedamzik 2004). From this it can be shown that $k_{I} \propto a^{-2 / 7}$, $M_{I} \propto a^{-8 / 7}$, and $\mathcal{H}_{I} \propto a^{2 / 7}$, where $a$ is the scale factor and $n=2$ is used in Eq. (2) due to causality constraints for the large scale spectrum (Banerjee \& Jedamzik 2004, Saveliev et al. 2012, 2013, Durrer \& Caprini 2003). Hence, the magnetic field strength on the integral scale evolves as

$$
B_{I} \propto \lambda_{I}^{-\frac{5}{2}} \text {. }
$$

Therefore, the above scaling due to the evolution decay law on the integral scale coincides with the scaling of the smoothed magnetic field in Eq. (2). The smoothed magnetic field $B_{L}$ on a scale $L$ is equivalent to the magnetic field on the integral scale $B_{I}$, assuming that most of the magnetic energy resides on the integral scale. The point is that, as the magnetic energy on small scales dissipate, the integral scale 
field $B_{I}$ moves down along the large scale spectrum, hence the above scaling.

Here we comment on the very interesting and exciting new developments in turbulent MHD where an apparent inverse transfer of magnetic energy occurs for the nonhelical case. Due to this effect the authors in (Kahniashvili et al. 2013) obtain a weaker evolution for non-helical magnetic fields $B_{I} \propto t^{-1 / 2}$ and $L_{I} \propto t^{1 / 2}$ giving the relation $B_{I} \propto \lambda_{I}^{-1}$ [c.f Eq. (9)] (see also (Campanelli 2004)). This numerically observed effect has also been studied in (Brandenburg et al. 2015) with the conditions of high resolutions, and magnetically dominant turbulence. However the condition of magnetically dominant turbulence is perhaps not satisfied in the early Universe. Magnetogenesis at first order phase transitions typically produce a lot of turbulent kinetic energy. The generated magnetic field, through dynamo action, comes into equipartition with the kinetic energy, but is unlikely to dominate over the kinetic energy e.g. (Sigl et al. 1997). Furthermore, in the study by (Brandenburg et al. 2015) it seems that the inverse transfer is less efficient for large Prandtl numbers, but the Prandtl numbers in the early Universe are huge. We clearly state here that for the following arguments we assume the decay laws of (Banerjee \& Jedamzik 2004, Campanelli 2007a, 2014) in Eq. (9), affirming that our assumptions could be challenged due to the important works of (Kahniashvili et al. 2013, Brandenburg et al. 2015).

If the helicity density is non-zero, the helicity fraction in the regime $f \ll 1$ evolves as $f=k_{I} \mathcal{H}_{I} / 8 \pi M_{I} \propto a^{8 / 7}$. This evolution occurs until the time of recombination, or until a state of maximum helicity is reached $f=1$, whichever comes first (Banerjee \& Jedamzik 2004, Saveliev et al. 2013). The evolution of the field strength and coherence length essentially ceases, i.e. with logarithmic scaling, when the Universe becomes matter dominated (Banerjee \& Jedamzik 2004). Following literature we approximate this time as the time of recombination.

When the maximal helicity $f=1$ is reached, an inverse cascade occurs where magnetic energy is transferred from small scales to large scales (Christensson et al. 2001). When applying helicity conservation $h_{B} \simeq$ const., remarkably the decay law becomes independent of the large scale slope and it can be shown that $k_{I} \propto a^{-2 / 3}, M_{I} \simeq$ const., $\mathcal{H}_{I} \propto a^{2 / 3}$, and the fraction remains constant $f=1$ (Christensson et al. 2001; Banerjee \& Jedamzik 2004, Saveliev et al. 2013). Hence, the field strength at the integral scale evolves as

$$
B_{I} \propto \lambda_{I}^{-\frac{1}{2}}
$$

which by coincidence is the same scaling as the Fermi observational bound in Eq. (6). This 'weaker' evolution of a maximally helical field in the radiation era can have important consequences for magnetic fields observed today.

We summarise the above results in Fig. 1 where the magnetic field evolves until recombination (index 'rec'), and the final field configuration falls on the line (Banerjee \&

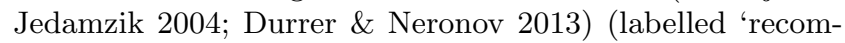
bination' in Fig. 1) $B_{\lambda, \text { rec }} / \mathrm{G} \simeq 8 \times 10^{-8} \lambda_{B \text {,rec }} / \mathrm{Mpc}$. This line corresponds to the largest eddies being processed at recombination $1 /\left.(a H)\right|_{\text {rec }} \simeq \lambda / v_{A}$ with $v_{A}$ the Alfvén speed (Jedamzik et al. 1998, Banerjee \& Jedamzik 2004). Hence, we obtain our first constraint. Magnetic fields generated during the radiation era will evolve to fall on the above line at recombination, which are also the values that will be observed today since the field strength and coherence length do not evolve significantly in the matter dominated Universe. In order for such fields to explain the Fermi observations it is required that $B_{\lambda, \text { rec }} \geqslant B_{\lambda, \text { rec }}^{\min }=4.3 \times 10^{-13} \mathrm{G}$ and $\lambda_{B \text {,rec }} \geqslant \lambda_{B, \text { rec }}^{\min }=5.4 \times 10^{-6} \mathrm{Mpc}$ (using the stronger bound in Eq. (6)). This minimum field configuration is labelled by point (a) in Fig. 1. From this minimum configuration we trace the evolution back to the time of magnetogenesis and find the minimum values for the field strength and coherence length at those times. We find that, if the field has zero initial helicity $f_{*}=0$, the initial field configuration must be (point (b) in Fig. 1 $B_{\lambda, *} \leqslant B_{\lambda, *}^{\max }=3 \times 10^{-6} \mathrm{G}$ and $\lambda_{B, *} \geqslant 9.9 \times 10^{-9} \mathrm{Mpc}$. This coherence length is smaller than the horizon size at the QCDPT $\sim 10^{-7} \mathrm{Mpc}$, but larger than the horizon size at the EWPT $\sim 10^{-10}$ Mpc. Hence, we come to our first important conclusion. With the stronger bound in Eq. (6), it is impossible to generate magnetic fields at the EWPT which can explain the apparently observed void magnetic fields if the magnetic fields have zero helicity.

To make this point even stronger we can ask the question: how far must the bound from $\gamma$-ray observations go down so that magnetic fields with zero helicity generated at the EWPT produce the void fields? Magnetic fields generated at the EWPT are constrained by (labelled by point (c) in Fig. 1 $B_{\lambda, *} \leqslant B_{\lambda, *}^{\max }=3 \times 10^{-6} \mathrm{G}$ and $\lambda_{B, *} \leqslant \lambda_{\mathrm{EW}}=2 \times 10^{-10}$ Mpc. With this we find that the new hypothetical "Fermi" constraint should be

$$
B_{\lambda} \gtrsim 1.5 \times 10^{-17}\left(\frac{B_{\lambda, *}}{B_{\lambda, *}^{\max }}\right)^{\frac{3}{7}}\left(\frac{\lambda_{B, *}}{\lambda_{\mathrm{EW}}}\right)^{\frac{15}{14}} \lambda_{B}^{-\frac{1}{2}} \mathrm{G} .
$$

This bound is compatible with the weakest constraint from $\gamma$-ray observations $B_{\lambda} \gtrsim 10^{-17}$ due to time delay suppression (Taylor et al. 2011) and the bound found in (Dermer et al. 2011) $B_{\lambda} \gtrsim 10^{-18}$. However, the expected coherence length of magnetic fields generated at the EWPT is roughly of order the bubble size, which is somewhat smaller than the horizon size by a factor $\beta \equiv \lambda_{B, *} / \lambda_{\mathrm{EW}} \sim 10^{-2}$ (Turok 1992). And, since $B_{\lambda, *}$ is expected to be a few orders of magnitude below $B_{\lambda, *}^{\max }$ (Baym et al. 1996), we can see that the existing Fermi bound would have to decrease considerably. Hence, even with the weakest constraints on void magnetic fields, fields generated at the EWPT most probably require helicity in order to explain the observed void fields.

Let us assume that magnetic fields are generated at the EWPT (Baym et al. 1996). If the initial helicity density is non-zero and the helicity fraction is large enough, then the inverse cascade may take effect and make it possible to explain the void fields. As seen above, when the spectral helicity is small, the fraction evolves as $f \propto a^{8 / 7}$ until a state of maximum helicity is reached $f_{\mathrm{tr}}=1$ at the transition time, therefore $f_{*}=\left(a_{*} / a_{\mathrm{tr}}\right)^{8 / 7}=\left(\lambda_{I, *} / \lambda_{I, \mathrm{tr}}\right)^{4}$. To find the minimum helicity fraction required in order to explain the Fermi observations, we note that $\lambda_{I, \text { tr }}$ falls on the Fermi constrain in Eq. (6) (labelled by point (d) in Fig. 1) since the Fermi constraint and the evolution for a maximally helical field has the same scaling $\lambda_{B}^{-1 / 2}$. From the above considerations we 


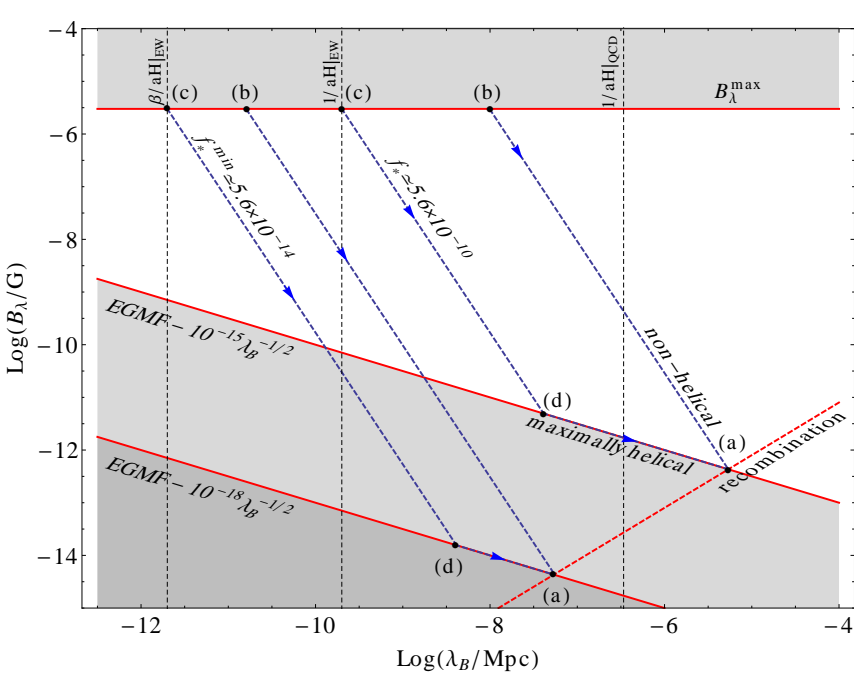

Figure 1. In the greyed out regions, constraints on present day magnetic fields are shown from Fermi observations of $\gamma$-ray sources (see text above Eq. (6) for the different constraints) and an upper bound set from energy considerations. Fields generated in the radiation era evolve to the line labelled "recombination". The evolutionary tracks from magnetogenesis until recombination are marked by dashed lines and depend on the helicity fraction $f_{*}$. The minimum field configuration at recombination to explain the void fields is marked by point (a). If there is zero helicity, the field configuration at megnetogenesis is marked by point (b). With non-zero helicity the initial field configuration can be reduced e.g point (c), where the field becomes maximally helical at point (d).

find that

$$
f_{*} \geqslant f_{*}^{\min }=\left(5.6 \times 10^{-10}\right) \frac{\lambda_{\mathrm{EW}}}{\lambda_{I, *}}\left(\frac{B_{\lambda, *}^{\max }}{B_{I, *}}\right)^{2},
$$

in order to explain the void magnetic fields. The above constraint is obtained using the stronger bound in Eq. (6), with the weaker bound and $\beta=10^{-2}$, the helicity fraction reduces to $f_{*}^{\min } \simeq 5.6 \times 10^{-14}$. We can also constrain the average helicity density, which is given by $h_{B} \simeq \rho k_{I} \mathcal{H}_{I}=8 \pi \rho f M_{I}=f B_{I}^{2} / k_{I}$. Since the helicity density is a conserved quantity $h_{B} \simeq$ const., we find that $h_{B, *} \simeq h_{B, \text { rec }}$. Hence, the minimum helicity density required to explain the Fermi observations is

$$
h_{B}^{\min } \simeq f_{*}^{\min } \frac{\lambda_{I, *}}{2 \pi} B_{I, *}^{2} \simeq 1.6 \times 10^{-13} \mathrm{nG}^{2} \mathrm{Mpc},
$$

which goes down to $h_{B}^{\min } \simeq 1.6 \times 10^{-19} \mathrm{nG}^{2} \mathrm{Mpc}$ when considering the weaker bound in Eq. (6) and $\beta=10^{-2}$.

In (Vachaspati 2001) the author estimated the primordial magnetic field helicity generated at electroweak baryogenesis. The production of baryon number requires changes in the Chern-Simons number, which are generated by the production and dissipation of nonperturbative field configurations, e.g. linked loops of electroweak strings. Such configurations would decay in the true vacuum phase of the EW transition leaving behind linked magnetic field lines. Hence the connection between baryon number and magnetic helicity. Indeed, the change in magnetic helicity is $\sim 10^{2}$ for every baryon produced (Vachaspati 2001). Hence, the helicity density can be estimated today as
$h_{B} \sim 10^{2} n_{b}$, where $n_{b} \sim 10^{-6} \mathrm{~cm}^{-3}$ is the baryon density observed today, therefore the helicity density is estimated as $h_{B} \sim 10^{-27} \mathrm{nG}^{2} \mathrm{Mpc}$, i.e. $f_{*} \sim 10^{-24}$ assuming $B_{\lambda, *}^{\max }$ and $\lambda_{\mathrm{EW}}$. The length scale in which helicity is expected to be maximal can be estimated by considering the characteristic length scale of the gauge field configurations $L \sim 1 / e^{2} T_{\mathrm{EW}}$ (Vachaspati 2001) which is much smaller than the horizon size at the EW scale by a factor $\sim 10^{-17}$. Therefore, with no other sources for generating magnetic helicity other than this simple mechanism from electroweak baryogenesis, we show that magnetic fields generated at the EWPT most probably cannot explain the void magnetic fields observed today. However, there are some exciting new ideas regarding Chiral MHD which can excitep helical magnetic fields at very hight temperatures (Boyarsky et al. 2012, 2015). It will be interesting to see how such mechanisms can affect our conclusions, this will be investigated in future publications.

\section{CONSTRAINTS FROM FIRST-ORDER PHASE TRANSITIONS}

The EWPT could be a first-order transition in certain extensions to the Standard Model (see e.g. (Laine \& Rummukainen 1998)). Such models can therefore be constrained by extragalactic magnetic fields, since their parameters, which characterise the phase transition, also determine the minimum helicity fraction required to produce the void fields.

Three parameters characterise model-independent analysis of first-order phase transitions (Steinhardt 1982, Espinosa et al. 2010). The first parameter $\alpha_{N} \equiv \epsilon_{\mathrm{vac}} / \rho_{\mathrm{rad}}$ is the ratio of the vacuum energy to the radiation energy density, which characterises the strength of the phase transition. The second is the efficiency parameter $\kappa \equiv u_{K}^{\text {bulk }} / \epsilon_{\mathrm{vac}}$, which defines the ratio of bulk kinetic energy over the vacuum energy. The third parameter is the bubble wall velocity $v_{b}$. It is shown that the efficiency parameter $\kappa$ depends on the bubble wall velocity $v_{b}$ and $\alpha_{N}$ (Steinhardt 1982, Kamionkowski et al. 1994: Espinosa et al. 2010). With equipartition between magnetic and kinetic energy, the fraction of magnetic energy to the radiation energy $f_{E}=u_{K} / \rho$ becomes $f_{E}=\kappa \alpha_{N}$, hence $\left(B_{\lambda, *}^{\max } / B_{I, *}\right)^{2}=1 / 2 \kappa \alpha_{N}$ in Eq. 12. Following the work of (Espinosa et al. 2010) we can explore the parameter space for $f_{*}^{\mathrm{mm}}$, independently of a specific particle physics model of the phase transition. The results are shown in Fig. 2. For example, for a weak phase transition $\alpha_{N}=0.01$ and subsonic bubble wall velocity $v_{b}=0.1$, we find the minimum helicity fraction $f_{*}^{\text {min }} \sim 10^{-3}$ at magnetogenesis. Whereas for a strong phase transition $\alpha_{N} \approx 1$ and supersonic bubble wall velocity $v_{b}=0.9$, we find the minimum helicity fraction $f_{*}^{\text {min }} \sim 10^{-8}$ at magnetogenesis i.e. much larger than the SM predictions from electroweak baryogenesis, where $\lambda_{I, *} / \lambda_{\mathrm{EW}} \sim 10^{-2}$ for the EWPT was used in both cases.

\section{CONCLUSIONS}

First-order phase transitions can generate magnetic fields in the early Universe. Under early Universe conditions with 




Figure 2. Depending on the phase transition parameters, i.e. the strength of the transition $\alpha_{N}$ and the bubble wall velocity $v_{b}$, the efficiency parameter $\kappa$ can be determined which in turn determines the energy of the phase transition that goes into producing magnetic fields. From this we can determine the minimum helicity fraction $f_{*}^{\text {min }}$ required to produce the void magnetic fields given a set of model parameters.

very small chemical potentials the QCDPT is a smooth transition (Aoki et al. 2006) whereas the EWPT could be firstorder in certain Standard Model (SM) extensions (Laine \& Rummukainen 1998). Inflationary magnetogenesis (Turner \& Widrow 1988), which is also beyond the SM, is another popular mechanism to explain void magnetic fields. Hence, the apparent observations of void fields from $\gamma$-ray observations seem to be a signature of physics beyond the SM or of new mechanisms which excite magnetic helicity (Boyarsky et al. 2012, 2015). If the constraints on void fields prove to be conclusive, then it is likely that magnetic helicity must play an important role. Here we show that magnetic fields generated at the EWPT must have significantly more helicity than that produced by electroweak baryogenesis in order to explain the extragalactic magnetic fields. To reach this conclusion we have assumed the magnetic decay laws of (Banerjee \& Jedamzik 2004, Campanelli 2007a 2014) and considered the simplest magnetic helicity estimates of (Vachaspati 2001). Our assumptions on the decay rates could be challenged due to new results of (Kahniashvili et al. 2013, Brandenburg et al. 2015.

\section{ACKNOWLEDGMENTS}

We thank G. Sigl and T. Konstandin for helpful discussions and comments. This work was supported by the Deutsche Forschungsgemeinschaft (DFG) through the collaborative research centre SFB 676 Particles, Strings, and the Early Universe.

This paper has been typeset from a $\mathrm{T}_{\mathrm{E}} \mathrm{X} / \mathrm{L}_{\mathrm{E}} \mathrm{X}$ file prepared by the author.

\section{REFERENCES}

Aoki Y., Endrodi G., Fodor Z., Katz S., Szabo K., 2006, Nature, 443, 675
Arlen T. C., Vassiliev V. V., Weisgarber T., Wakely S. P., Shafi S. Y., 2012

Banerjee R., Jedamzik K., 2004, Phys.Rev., D70, 123003

Baym G., Bodeker D., McLerran L. D., 1996, Phys.Rev., D53, 662

Biskamp D., 1993, Nonlinear magnetohydrodynamics

Boyarsky A., Frohlich J., Ruchayskiy O., 2012, Phys. Rev. Lett., 108, 031301

Boyarsky A., Frohlich J., Ruchayskiy O., 2015

Brandenburg A., Kahniashvili T., Tevzadze A. G., 2015, Phys.Rev.Lett., 114, 075001

Broderick A. E., Chang P., Pfrommer C., 2012, Astrophys. J. , 752, 22

Campanelli L., 2004, Phys.Rev., D70, 083009

Campanelli L., 2007a, Phys.Rev.Lett., 98, 251302

Campanelli L., 2007b, Phys.Rev.Lett., 98, 251302

Campanelli L., 2014, Eur.Phys.J., C74, 2690

Christensson M., Hindmarsh M., Brandenburg A., 2001, Phys.Rev., E64, 056405

Dermer C. D., Cavadini M., Razzaque S., Finke J. D., Chiang J., Lott B., 2011, ApJ, 733, L21

Dolag K., Kachelriess M., Ostapchenko S., Tomas R., 2011, Astrophys.J., 727, L4

Durrer R., Caprini C., 2003, JCAP, 0311, 010

Durrer R., Neronov A., 2013, Astron.Astrophys.Rev., 21, 62

Espinosa J. R., Konstandin T., No J. M., Servant G., 2010, JCAP, 1006, 028

Essey W., Ando S., Kusenko A., 2011, Astropart.Phys., 35, 135

Jedamzik K., Katalinic V., Olinto A. V., 1998, Phys.Rev., D57, 3264

Kahniashvili T., Tevzadze A. G., Brandenburg A., Neronov A., 2013, Phys.Rev., D87, 083007

Kahniashvili T., Tevzadze A. G., Ratra B., 2011, Astrophys.J., 726, 78

Kamionkowski M., Kosowsky A., Turner M. S., 1994, Phys.Rev., D49, 2837

Laine M., Rummukainen K., 1998, Nucl.Phys., B535, 423

Neronov A., Vovk I., 2010, Science, 328, 73

Saveliev A., Jedamzik K., Sigl G., 2012, Phys.Rev., D86, 103010

Saveliev A., Jedamzik K., Sigl G., 2013, Phys.Rev., D87, 123001

Sigl G., 2002, Phys.Rev., D66, 123002

Sigl G., Olinto A. V., Jedamzik K., 1997, Phys.Rev., D55, 4582

Steinhardt P. J., 1982, Phys.Rev., D25, 2074

Tashiro H., Chen W., Ferrer F., Vachaspati T., 2013

Tavecchio F., Ghisellini G., Foschini L., Bonnoli G., Ghirlanda G., et al., 2010, Mon.Not.Roy.Astron.Soc., 406, L70

Taylor A., Vovk I., Neronov A., 2011, Astron.Astrophys., 529, A144

Turner M. S., Widrow L. M., 1988, Phys.Rev., D37, 2743

Turok N., 1992, Phys.Rev.Lett., 68, 1803

Vachaspati T., 2001, Phys.Rev.Lett., 87, 251302

Wagstaff J. M., Banerjee R., Schleicher D., Sigl G., 2014, Phys.Rev., D89, 103001 